\title{
Effects on SuperDARN HF radar echoes of sudden impulses of solar wind dynamic pressure
}

\author{
I. Coco ${ }^{1,2}$, E. Amata ${ }^{1}$, M. F. Marcucci ${ }^{1}$, M. De Laurentis ${ }^{3}$, J. P. Villain ${ }^{4}$, C. Hanuise ${ }^{4}$, and M. Candidi ${ }^{1}$ \\ ${ }^{1}$ Istituto di Fisica dello Spazio Interplanetario (IFSI), INAF, Rome, Italy \\ ${ }^{2}$ Earth Science Dept., University of Siena, Italy \\ ${ }^{3}$ Vitrociset S.p.a., Rome, Italy \\ ${ }^{4}$ Laboratoire de Physique et Chimie de l'Environnement (LPCE), CNRS/Université d'Orléans, Orléans, France
}

Received: 4 August 2004 - Revised: 9 May 2005 - Accepted: 17 May 2005 - Published: 28 July 2005

\begin{abstract}
In this work we perform a statistical analysis of the ionospheric echo response observed by six radars of the SuperDARN network in the Northern Hemisphere, over 236 Sudden Impulses (SI) of solar wind dynamic pressure events (from 1997 through 2000). For that purpose, we make use of MRS, the Mean Rate of Scattering, as a function of time during the SI event. We classify the events in sudden increases (I events, 144 cases) and decreases (D events, 92 cases) of the solar wind dynamic pressure. Moreover, we make use of the $\mathrm{AE}$ index to define two distinct conditions of the ionosphere under which each event may take place: Quiet and Disturbed. Regarding Quiet conditions, for both I and D events, we find that MRS displays an increase related to the SI time. On the contrary, for Disturbed conditions, D events display an increase in MRS, while I events show a clear dip. The similarity of response for I and D events under Quiet conditions is briefly discussed, but the smaller number of $\mathrm{D}$ events does not allow one to further analyse them.
\end{abstract}

As for the I events, a latitudinal analysis shows that the MRS increase for Quiet conditions is seen both at low latitudes $\left(60^{\circ}-70^{\circ} \Lambda\right)$ and at high latitudes $\left(70^{\circ}-80^{\circ} \Lambda\right)$; for Disturbed Is the MRS decrease is stronger at high latitudes. We suggest that the MRS increase for Quiet Is can be due to two different mechanisms: 1) a soft electron precipitation induced by Field Line Resonances (FLR) or loss cone instability at lower latitudes; 2) an enlargement of the cusp at higher latitudes, which in turn may induce enhanced particle precipitation.

For what concerns Disturbed Is, the MRS decrease can be produced by a higher energy electron precipitation $(>1 \mathrm{keV})$, that enhances the electron density in the $\mathrm{E}$ and $\mathrm{D}$ regions. This provokes a strong absorbtion of the radio waves in the $\mathrm{D}$ region and a higher refraction in the $\mathrm{E}$ region, leading to a decrease in MRS, especially at higher latitudes.

Correspondence to: I. Coco

(igino.coco@ifsi.rm.cnr.it)
For I events a further classification has been made on the basis of IMF orientation: this suggests that the effects summarized above are due to the SI itself.

Keywords. Ionosphere (Ionospheric irregularities) - Magnetospheric physics (Magnetosphere-ionosphere interactions; Solar wind-magnetosphere interactions)

\section{Introduction}

Sudden Impulses of solar wind dynamic pressure (SI) have long been known to trigger a global response of the magnetosphere-ionosphere system. SIs are sudden variations of the solar wind dynamic pressure associated with corotating and travelling solar wind shocks and tangential discontinuities, whose effects on the magnetosphere extend from hours to days (Sibeck, 1990, and ref. therein). A pressure perturbation generates a compressional MHD wave which will propagate in the magnetospheric cavity. Coupling to the ionosphere requires the generation of a Field-Aligned Current (FAC) system that is carried by a field-guided Alfvén mode, thus leading to the formation of characteristic vortex-like structures, whose signatures are well identified in ground magnetometers (e.g. Friis-Christensen et al., 1988; Araki, 1994; Sibeck et al., 2003. For a discussion on the MHD modes formation and coupling, see Southwood and Kivelson, 1990). Following Araki (1994), both positive variations (sudden compressions of the magnetopause), and negative variations (sudden relaxations of the magnetopause) of the solar wind dynamic pressure, should act on the magnetosphere-ionosphere system in the same way, but for the FAC direction and the vortex circulation sense, which should be opposite in the two cases. Recently, Takeuchi et al. (2000) made a case study of a negative sudden impulse, and found a good agreement with Araki's picture. Thorolfsson et al. (2001) reported about two SIs, one positive and one negative, with a few hours separation. 


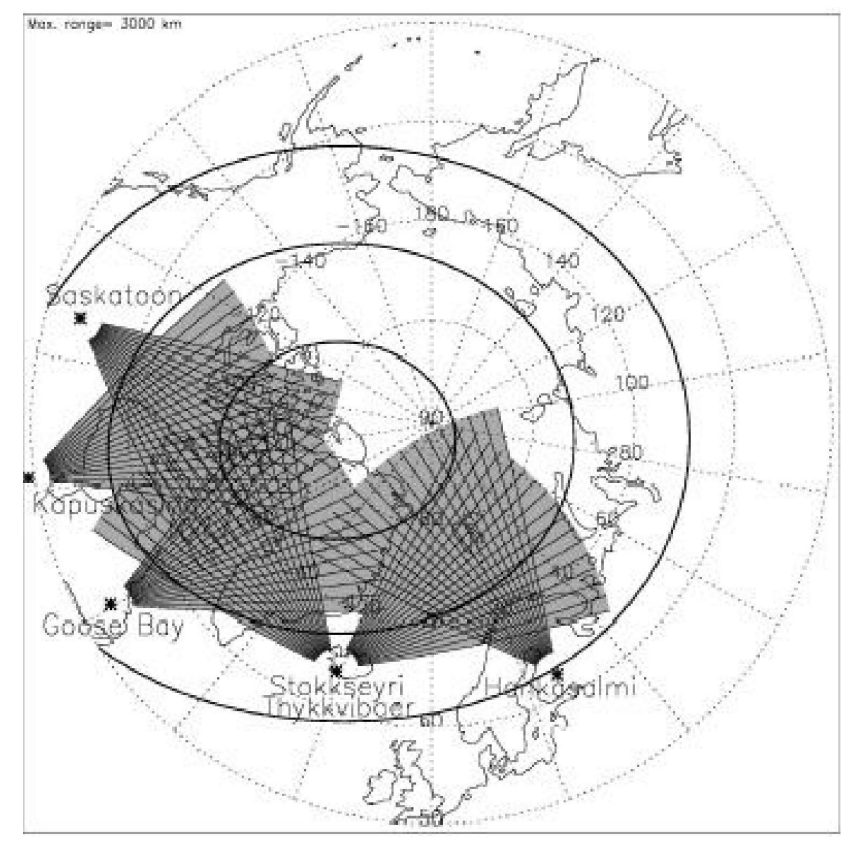

Fig. 1. Location and fields of view of the SuperDARN radars used in this study. The thick line contours mark $60^{\circ}, 70^{\circ}$ and $80^{\circ}$ Invariant Latitudes.

They made a comparison between GOES-8 magnetic field, NOAA-14 particle precipitation, Greenland magnetometer chain data and plasma convection velocity in the ionosphere determined through the SuperDARN Stokkseyri radar. The radar clearly showed bursts of enhanced velocity, whose direction and orientation were in agreement with vortices formation as predicted by Araki (1994). Zhou and Tsurutani (1999), through the observation of two positive SIs, suggest that the pressure pulse, squeezing the magnetopause from the nose to the flanks, modifies the field lines geometry and leads to an increase in the perpendicular kinetic energy of the trapped particles. This results in a loss cone instability, with excitation of whistler modes and enhancement of pitch angle scattering, thus inducing particle precipitation in the ionosphere. Many other case studies have been published (e.g. Russell et al., 1994; Liou et al., 2002); nevertheless, very few statistical works on SIs have been done. Sitar et al. (1996) selected 27 pressure increases and 16 pressure decreases of solar wind dynamic pressure, and examined the statistical response of the east-west component of the geomagnetic field, as measured by the magnetometers of the Greenland chain. They found that the ground response does not consistently conform to existing theoretical models of field-aligned currents generated by changes in dynamic pressure. To this respect the authors give several caveats, for example, the fact that the magnetometers are highly sensitive to local features and perturbations in the ionosphere, and that the filamentary FAC induced by a SI could not have enough energy to produce a Hall current system strong enough, in order to be detected by a magnetometer.
In this study, we investigate possible global effects of SIs on the amount of backscatter from SuperDARN radars over a large set of events. Radar echoes, in fact, come from density gradients associated with plasma instabilities in the ionosphere, which can be affected by enhanced precipitation levels, for example, as described by Zhou and Tsurutani (1999).

In Sect. 2 we describe the data set. The analysis method is detailed in Sect. 3, while Sect. 4 is devoted to the full description of the results. The work is then completed by a discussion and a summary in Sects. 5 and 6.

\section{Description of the data set}

The present work is based on data collected by the following six coherent SuperDARN HF radars in the Northern Hemisphere: Hankasalmi, Thykkvibær, Stokkseyri, Goose Bay, Kapuskasing and Saskatoon. AE index and WIND data have also been used for classification purposes. The principle of operation of SuperDARN is fully described in Greenwald et al. (1995). Here we shall only recall that the radars operate synchronously and continuously in a frequency range $8-20 \mathrm{MHz}$, and record the backscattered signals from the ionosphere. Figure 1 shows the fields of view of the radars listed above: each one covers $52^{\circ}$ in azimuth, through 16 discrete beams, while the echoes are sorted in $45-\mathrm{km}$ range gates, from $180 \mathrm{~km}$ to $3500 \mathrm{~km}$ from the radar. Data are acquired in 2-min scans, all starting at even minutes. From the complex autocorrelation function of the backscattered signals, it is possible to derive the horizontal ambient plasma velocity along the line of sight, the spectral width of the measured velocity and the Signal-to-Noise Ratio (SNR) (e.g. Hanuise et al., 1985; Villain et al., 1987). Signals reflected twice in the ionosphere and backscattered from the ground are known as groundscatter. They are characterized by very low velocities and spectral widths, and have been excluded from the analysis described herein. The WIND Magnetic Field and Solar Wind Experiments are described by Lepping et al. (1995) and Ogilvie et al. (1995). The provisional AE data used herein have been obtained from the World Data Center for Geomagnetism, Kyoto (http://swdedb.kugi. kyoto-u.ac.jp/aedir/).

All the summary plots of WIND data from November 1997 to September 2000 were visually inspected and 236 SI events were identified, 144 Increases (I) of solar wind dynamic pressure and 92 Decreases (D), meeting the following criteria:

1. Solar wind dynamic pressure roughly constant over at least one hour prior to the SI,

2. $|\Delta p|>3 \mathrm{nPa}$, where $\Delta p$ is the jump (positive or negative) in pressure,

3. $\Delta t \leq 10 \mathrm{~min}$, where $\Delta t$ is the rise (fall) time of the pressure jump. 
For each event we calculate a delay between the WIND event time, $T_{W I N D}$, and the expected arrival time on the ground simply as:

$\Delta \tau=\frac{X}{v_{x}}$,

where $X$ is the $x$ component of the WIND position in GSM coordinates, and $v_{x}$ is the corresponding solar wind velocity component. On this basis we defined an expected event time on the ground as $T_{g}=T_{W I N D}+\Delta \tau$. For each event we built a database comprising of:

- One hour of WIND plasma parameters (pressure, density and velocity with 90 -s resolution) and IMF data (with 1-min resolution) centered on $T_{W I N D}$,

- One hour of AE index (with 1-min resolution) data centered on $T_{g}$,

- One hour of SuperDARN data (with 2-min resolution), also centered on $T_{g}$, from the six listed radars.

In the present study we make use of a simple parameter to measure the amount of backscatter recorded by a radar as a function of time during each one hour period. For each event we define a time interval spanning from -30 to $30 \mathrm{~min}$ about a central time $T=0$, corresponding to $T_{W I N D}$ for the wind data and to $T_{g}$ for the ground-based data. This time range is then divided into 15 negative and 15 positive 2-min bins, each one corresponding to a complete radar scan. Let us consider a 5-dimensional matrix $n\left(e v, R, b, r, t_{i}\right)$, where $e v$ and $R$ refer to a given event and radar, $b$ and $r$ to a given beam and range, and $t_{i}$ is the center of a two-minute bin. The matrix elements are set to 1 if its echo is not classified as ground scatter and has a SNR greater than $6 \mathrm{~dB}$; otherwise, they are set to 0 . In a statistical study over a long time period, Ruohoniemi and Greenwald (1997) set a threshold of $3 \mathrm{~dB}$; on the other hand, Ballatore et al. (2001) used a threshold of $6 \mathrm{~dB}$. In our case, since the data set is considerably smaller, we chose a $6-\mathrm{dB}$ threshold to sort relatively stronger signals, possibly independent of instrumental/noise effects.

Figure 2 shows an SI event from our data set, which was observed by WIND at $T_{W I N D}=21: 00$ UT on 20 February 2000. From top to bottom, the three panels display as a function of time: the solar wind dynamic pressure, the AE geomagnetic index, and $n_{e v, R}^{\prime}\left(t_{i}\right)$ for the Goose Bay (solid line) and Kapuskasing radars (dashed line), where

$n_{e v, R}^{\prime}\left(t_{i}\right)=\sum_{b} \sum_{r} n\left(e v, R, b, r, t_{i}\right)$.

The time axis ranges from -30 to 30 min around the central $T=0$ event time, which corresponds to $T_{W I N D}$ for the top panel and to $T_{g}$ for the middle and bottom panels. This same time axis shall be used in all figures hereafter.

Prior to the SI, the solar wind pressure was close to $2 \mathrm{nPa}$, exhibiting only small fluctuations. At $T=0$ the pressure jumped to more than $6 \mathrm{nPa}$ and decreased slowly between $T \simeq 16$ and $T \simeq 30$ min to about $5 \mathrm{nPa}$. The AE index displays

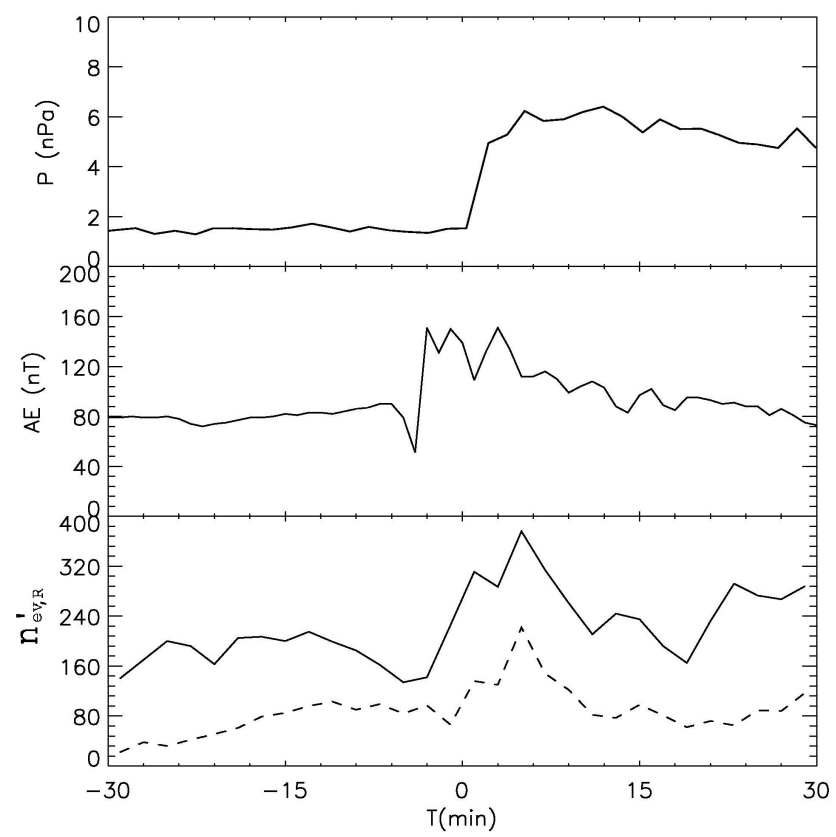

Fig. 2. SI event observed by WIND at 21:00 UT on 20 February 2000. From top to bottom: solar wind dynamic pressure measured by WIND, geomagnetic AE index, number of echoes $n_{e v, R}^{\prime}$, summed over all beams and ranges for each two-min bin, for the Goose Bay (solid curve) and Kapuskasing (dashed curve) radars. The time axis ranges from -30 to $30 \mathrm{~min}$ around the central $T=0$ event time, which corresponds to $T_{W I N D}$ for the top panel and to $T_{g}$ for the middle and bottom panels.

a sharp bipolar pulse at $T \simeq-5 \mathrm{~min}$, resulting in a net increase by $70 \mathrm{nT}$, followed by some oscillations lasting $8 \mathrm{~min}$ and a slow decrease over $30 \mathrm{~min}$ to the pre-SI level. The Goose Bay and Kapuskasing number of echoes undergoes a 10-min broad peak starting at $T=0$, possibly preceded by a dip starting a few minutes before. We interpret the AE and SuperDARN response as a result of the SI observed at WIND. If this is so, the timing of the observations implies that the 43min time shift applied through Eq. (1) to the ground-based data is overestimated by at least $5 \mathrm{~min}$. The example of Fig. 2 shows that the delay calculated as in Eq. (1) is subject to significant errors. We will devote part of the discussion (Sect. 5) to this point.

In order to have a first direct insight into the statistical significance of the data set, all the echoes were sorted according to magnetic latitude $(\Lambda)$, and MLT. The $\Lambda \times$ MLT space is divided into cells of $2^{\circ}$ in magnetic latitude, between $50^{\circ}$ and $90^{\circ} \Lambda$, and one hour in MLT. For each such cell we then calculate the total number of echoes, $\mathrm{N}(\Lambda$, MLT), by summing over all radars, all events and time bins. Figure 3 displays the resulting $\Lambda \times$ MLT plot, in logarithmic units. We notice that most echoes are concentrated between $64^{\circ}$ and $68^{\circ} \Lambda$, with a minimum around $10: 00 \sim 11: 00$ MLT and a maximum around 23:00 MLT. The backscattered signal is also seen at higher latitude, between $72^{\circ}$ and $76^{\circ} \Lambda$, limited to the dayside, and corresponding well to the ionospheric footprint of 


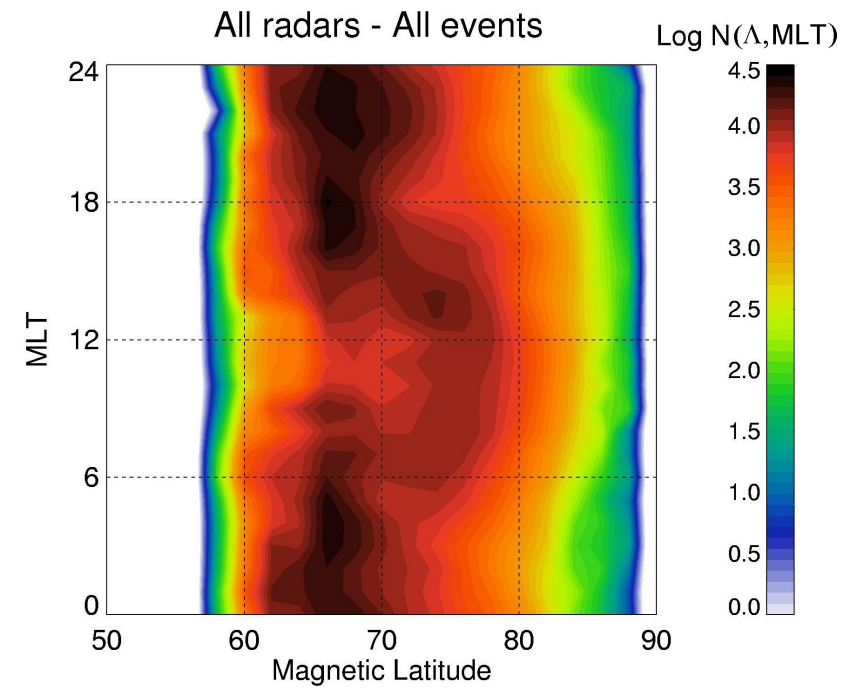

Fig. 3. Isocontours of the number of echoes summed over all radars, events and two-min bins, in cells of $2^{\circ}$ Magnetic Latitude, $\Lambda(x$ axis), and one hour MLT ( $y$ axis). The log-number of echoes is coded according to the color scale on the right.

the cusp region (Newell and Meng, 1992). These characteristics are consistent with other statistical works that made use of larger SuperDARN databases (Milan et al., 1997; Ruohoniemi and Greenwald, 1997; Ballatore et al., 2001; Villain et al., 2002).

\section{Analysis method}

We define the percent Rate of Scattering (RS) for one event, as a function of $t_{i}$, as:

$\operatorname{RS}\left(t_{i}, e v\right)=\frac{\sum_{R} \sum_{b} \sum_{r} n\left(e v, R, b, r, t_{i}\right)}{n_{\text {tot }}(e v)} \times 100$,

where $n_{\text {tot }}(e v)=\sum_{R} \sum_{b} \sum_{r} \sum_{i} n\left(e v, R, b, r, t_{i}\right)$ is the total number of echoes for an event $e v$. Other authors have calculated RS in different ways. For example, Ballatore et al. (2001) calculated RS as in Eq. (3), but used 6-min bins; moreover, they subtracted an average daily modulation, calculated over one-month intervals. On the contrary, the subtraction of such a modulation is not necessary in the present study, as Rates of Scattering are averaged over various event subsets to evidence the characteristic response to SIs pertaining to different conditions. For a given set of events, we call $N_{e v}$ the number of events and define the Mean Rate of Scattering, MRS, the variance $\sigma_{R}$ of the echo distribution and the statistical error, $\epsilon$, as a function of $t_{i}$ :

$\operatorname{MRS}\left(t_{i}\right)=\frac{\sum_{e v} \operatorname{RS}\left(t_{i}, e v\right)}{N_{e v}}$,

$\sigma_{R}\left(t_{i}\right)=\sqrt{\frac{\sum_{e v}\left(\operatorname{RS}\left(t_{i}, e v\right)-\operatorname{MRS}\left(t_{i}\right)\right)^{2}}{N_{e v}-1}}$,

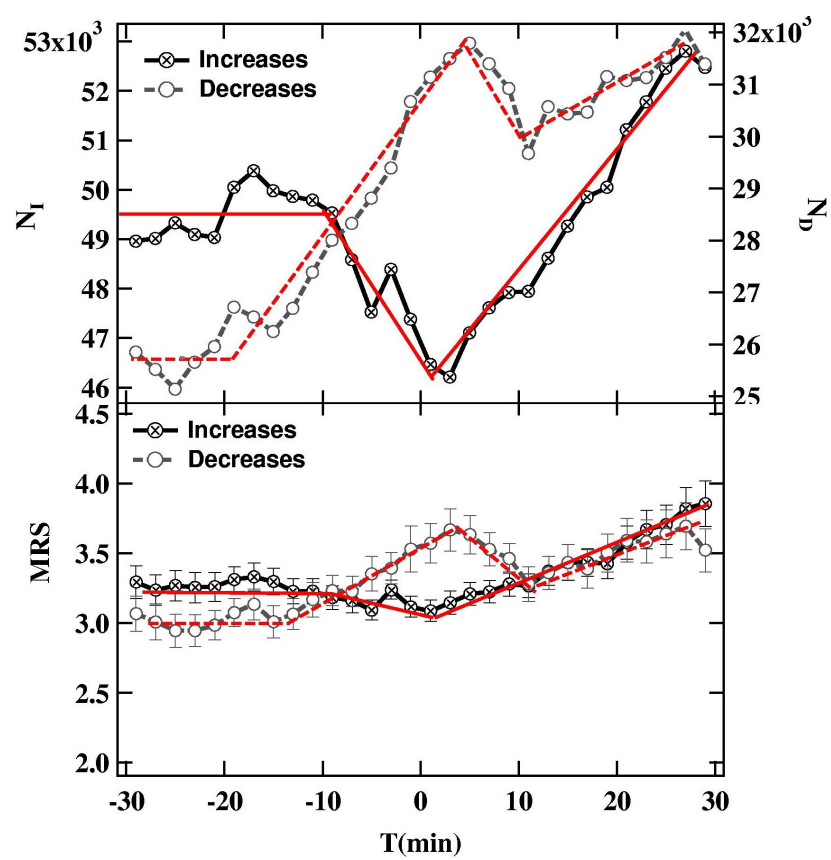

Fig. 4. The upper panel shows the number of echoes vs. time for Increases (114 events, black curve, $N_{I}$, left axis) and Decreases (64 events, grey curve, $N_{D}$, right axis). The lower panel shows MRS vs. time for I (black curve) and D (grey curve) events. The red lines superimposed on the curves highlight the main trends.

$\epsilon=\frac{\sigma_{R}\left(t_{i}\right)}{\sqrt{N_{e v}}}$.

By examining the two ensembles of 144 Is and 92 Ds, defined in Sect. 2, one sees that a certain number of events has very few echoes. According to Eqs. (4), (5) and (6), such events lower MRS and enhance the variance. On the other hand, their contribution to the total amount of scatter is negligible. Therefore, as a compromise between the necessity to have large statistics, and to clean MRS and variances from statistical noise, we choose to remove the events with $n_{\text {tot }}(e v)<1000$. This seems reasonable if one considers that the total number of available cells for each event is 216000 (6 radars, 16 beams, 75 ranges, 30 time bins). In the following analysis we then proceed with a reduced data set of 178 events: 114 Is and 64 Ds.

\section{Description of the results}

This study is aimed at evidencing the global main effects of SIs on the backscattered signal. Therefore, in all the plots shown hereafter we will concentrate on macroscopic and general features. To that purpose, red lines have been superimposed on the curves, in order to focus the attention on their main features and trends, neglecting variations of the order of the error bars.

In Fig. 4 the number of echoes and the MRS are plotted against time for I and $\mathrm{D}$ events. The upper panel displays the number of echoes for I $\left(N_{I}\right.$, black curve) and $\mathrm{D}\left(N_{D}\right.$, grey 
curve) events. The I curve (114 events, 1497602 echoes) is roughly constant up to $T \simeq-10 \mathrm{~min}$, when a decrease is observed, reaching a minimum at $T \simeq 4 \mathrm{~min}$. In the last part of the interval the curve rises almost monotonically. The D curve (64 events, 873154 echoes), on the contrary, starts to rise at $T \simeq-18 \mathrm{~min}$ and reaches a maximum at $T \simeq 4 \mathrm{~min}$. Then one notices a fall to $T \simeq 12 \mathrm{~min}$ and again a rise of the trend in the last part of the interval. The lower panel displays the MRS; error bars calculated from Eq. (6) are included. The behaviour of MRS for Is is nearly flat in the first part of the interval; then, at $T \simeq-15 \mathrm{~min}$, MRS begins to decrease, with a minimum at $T \simeq 2 \mathrm{~min}$. In the second part of the interval, MRS rises almost continuously, reaching a higher level than in the beginning. On the other hand, the $\mathrm{D}$ curve starts to rise at about $T \simeq-15 \mathrm{~min}$, reaches a maximum at $T \simeq 4 \mathrm{~min}$, then decreases up to $T \simeq 12 \mathrm{~min}$ and finally rises again, similar to the I curve. The broad peak observed for the D curve is affected by large error bars in the $[-5,5]$ min interval, while for the I curve the error bars are generally lower.

As Fig. 4 refers to data for all events and all radars, the variations described with time of the number of echoes and MRS may result from the superposition of effects depending on various conditions in the ionosphere, in the magnetosphere and in the solar wind; therefore, it is reasonable to group the events into different subsets, to try to evidence the echo response for each of them. The most relevant parameters to group the events are the AE index, IMF orientation, MLT and magnetic latitude. Therefore, in Subsects. 4.1 through 4.3 the data are sorted accordingly.

From the description of Fig. 4, it appears that $N$ and MRS display essentially the same features as a function of time. From now on we will only show MRS plots which allow one to assign to each point its statistical error, as defined in Eq. (6).

\subsection{AE index analysis}

Visual inspection of plots of solar wind parameters and MRS for all the events suggested to us that some MRS variations might not be directly triggered by the SI. The natural interpretation for such cases is that MRS responds to changes in the ionospheric conditions of internal origin. It is therefore useful to include in our analysis a parameter to describe the state of the ionosphere in the regions under study prior to the SI. For that purpose we decided to make use of the Auroral Electrojet index (AE), introduced by Davis and Sugiura (1966), to monitor the occurrence of auroral phenomena, and more generally magnetospheric substorms. AE is derived from high-latitude fluctuations of the magnetic field horizontal component at the Earth's surface and is meant to estimate the total maximum intensity of the ionospheric auroral current system.

We decided to group the events into two classes, Quiet and Disturbed events, defined on the basis of the AE index, and to further pursue the analysis on each class separately. Let $M=\langle A E\rangle+\sigma$, and $Q=(2 \sigma) /\langle A E\rangle$, where $\langle A E\rangle$ is the time average of $\mathrm{AE}$ and $\sigma$ its standard deviation calculated over a $[-30,10]$ min interval about $T_{g}$. To this regard, a natural choice would have been the $[-30,0]$ min interval; however, we extended it by $10 \mathrm{~min}$ to allow for the uncertainty in the $T_{g}$ calculation. We define:

- Quiet event. An event which satisfies one of the following conditions:

1. $M<200 \mathrm{nT}$,

2. $200 \leq M \leq 300 \mathrm{nT}$, and $Q \leq 0.5$.

- Disturbed event. An event which satisfies one of the following conditions:

1. $M>400 \mathrm{nT}$,

2. $300 \leq M \leq 400 \mathrm{nT}$, and $Q$ geq 0.5 .

- Intermediate event. An event which satisfies one of the following conditions:

1. $200 \leq M \leq 300 \mathrm{nT}$, and $Q>0.5$,

2. $300 \leq M \leq 400 \mathrm{nT}$, and $Q<0.5$.

The Intermediate class has the purpose of clearly separating the Quiet and Disturbed events. Consequently, Intermediate events, 14 in number, are excluded from the following analysis.

Figure 5 shows three examples of events, one for each class defined above. The solid and dotted lines refer respectively to AE (axis on the left) and to the dynamic pressure (axis on the right). The upper panel shows an example of Quiet event which meets the condition $M<200 \mathrm{nT}$. The middle panel shows an Intermediate event for which $300 \leq M \leq 400 \mathrm{nT}$, and $Q<0.5$. The lower panel shows a Disturbed event which meets the condition $M>400 \mathrm{nT}$.

Figure 6 displays MRS against time for the two subsets of Quiet and Disturbed events, for both Is and Ds. In the upper panel, which refers to Quiet events (60 I and 29 D), the curve for Is (black line) starts with a rough plateau and displays a positive trend starting around $T=-4 \mathrm{~min}$; the curve for Ds (grey line) displays larger fluctuations, nevertheless, it is possible to recognize two levels beyond the error bar uncertainty: one lower, in the first part of the plot $(-28<T<-23 \mathrm{~min})$, and one higher, at the end $(20<T<30 \mathrm{~min})$. The lower panel of Fig. 6 shows the results for Disturbed events (48 I and $27 \mathrm{D}$ ): the I curve (black) shows a strong decrease lasting from $T \simeq-20 \mathrm{~min}$ up to $T \simeq 2$ min, while the last part of the plot is almost flat with some fluctuations. This suggests that the depletion of echoes for Is, already seen in the whole data set in Fig. 4, is due to the Disturbed events. For the D curve the presence of large fluctuations does not allow one to unambiguously distinguish between two different levels.

As already seen in the lower panel of Fig. 4, again the $\mathrm{D}$ curves display larger error bars; moreover, D events are only 56 (compared to 108 Is), which implies a substantially smaller statistical significance for their subset. As in the following paragraphs the events shall be further classified into subsets according to IMF, MLT and magnetic latitude, we limit such analysis to I events. 


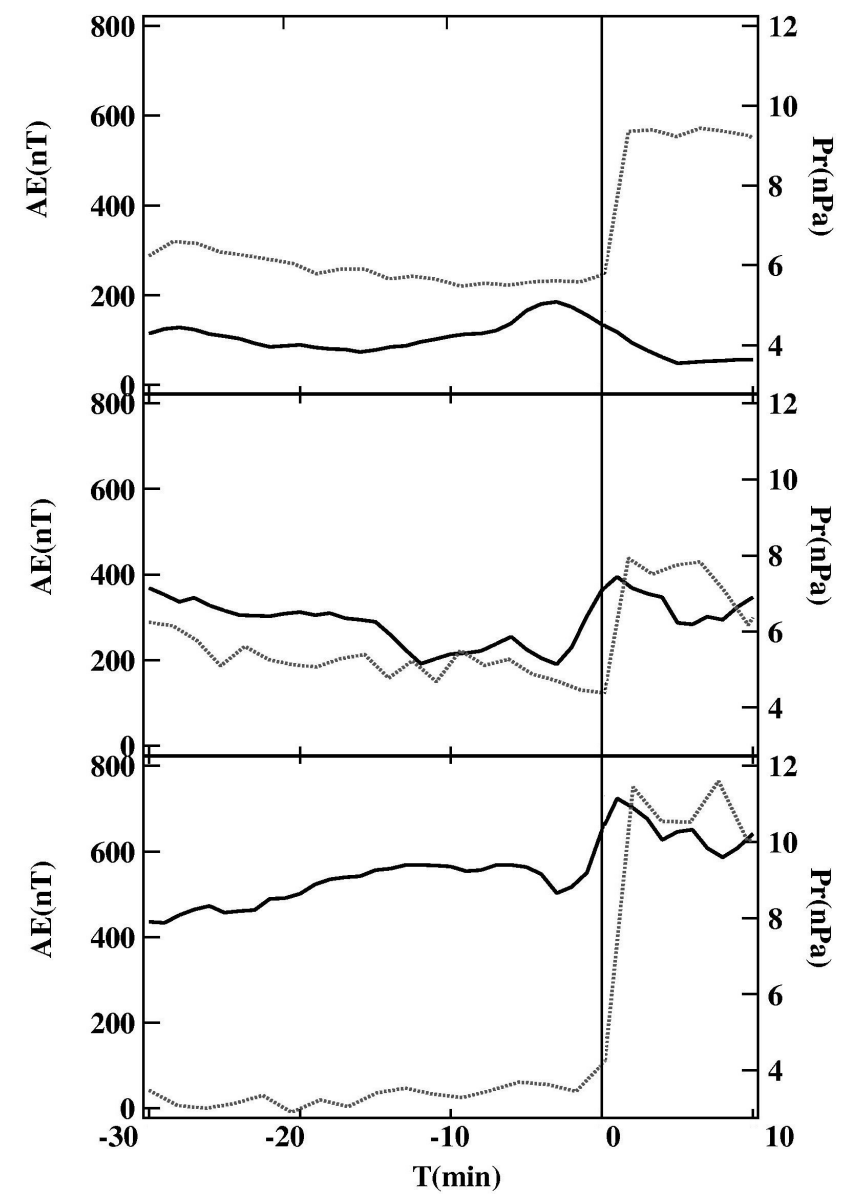

Fig. 5. Upper panel: example of Quiet event $\left(T_{W I N D}=06: 15\right.$ UT on 1 November 1997. $T_{g}=06: 43$ UT). Middle panel: example of Intermediate event $\left(T_{W I N D}=10: 10\right.$ UT on 10 December 1997. $T_{g}=11: 23$ UT). Lower panel: example of Disturbed event ( $T_{W I N D}=18: 10$ UT on 1 August 1998. $T_{g}=18: 31 \mathrm{UT}$ ). In all panels the solid curves refer to AE (vertical axis on the left), while the dotted ones refer to the solar wind dynamic pressure (vertical axis on the right). On the horizontal axis $T=0$ is $T_{g}$ for AE and $T_{W I N D}$ for the pressure.

\subsection{IMF analysis}

This paragraph is devoted to study MRS as a function of the IMF orientation, always keeping the previously described Quiet/Disturbed classification. First of all, we checked that the MRS behaviours so far described can be attributed to the SI itself. In order to do that, we visually inspected all IMF plots looking for IMF direction changes and made the following classification of the events:

- $B_{z}^{(+)}$: events for which $B_{z} \geq 0$ in a time interval $[-30,10]$ min with respect to $T_{W I N D}$,

- $B_{z}^{(-)}$: events for which $B_{z} \leq 0$ in a time interval $[-30,10]$ min with respect to $T_{W I N D}$,

- $B_{z}^{v a r}$ : events which display at least a $B_{z}$ polarity reversal in the $[-30,10]$ min interval.

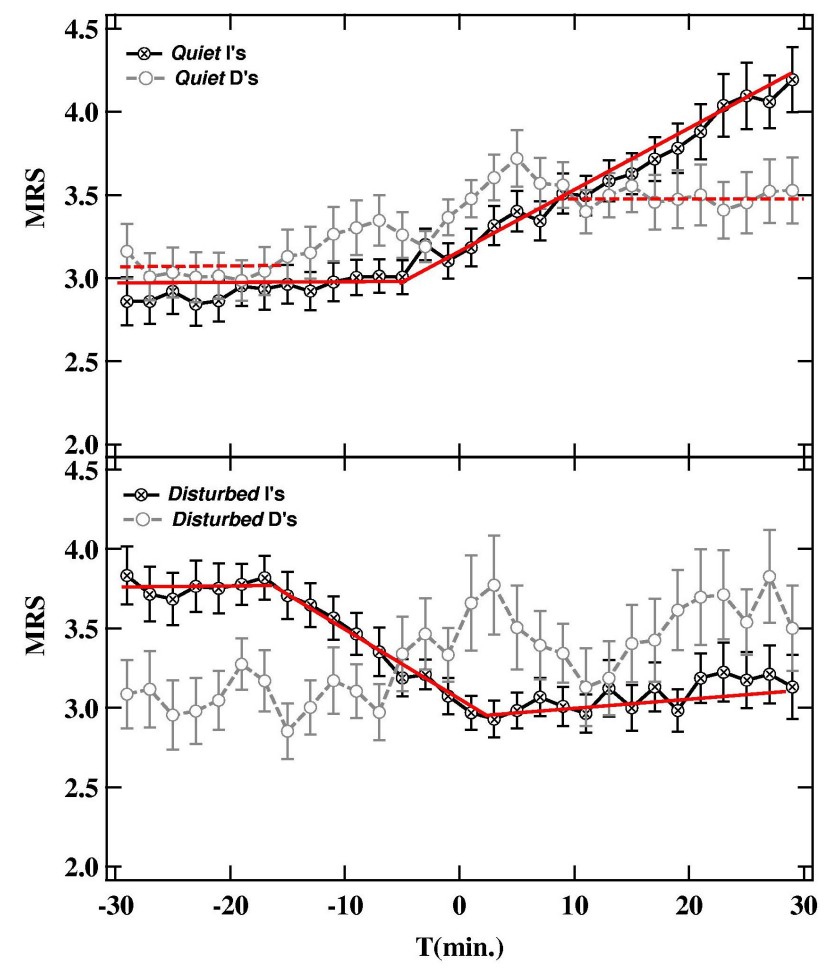

Fig. 6. Upper panel: plot of MRS vs. time for Increases (black curve) and Decreases (grey curve) for Quiet events (60 I, 29 D). Lower panel: the same as for the upper panel, but for Disturbed events (48 I, $27 \mathrm{D})$.

Table 1. Number of Quiet and Disturbed Is as for the $B_{z}^{(+)}, B_{z}^{(-)}$ and $B_{z}^{v a r}$ subsets, i.e. events for which $B_{z}$ is positive, negative or variable in a $[-30,10]$ min interval about $T_{W I N D}$.

\begin{tabular}{cccc}
\hline Type & Tot & Quiet & Disturbed \\
\hline$B_{z}^{(+)}$ & 38 & 35 & 3 \\
$B_{z}^{(-)}$ & 48 & 13 & 35 \\
$B_{z}^{\text {var }}$ & 22 & 12 & 10 \\
\hline
\end{tabular}

The associated statistics are reported in Table 1 . We then plotted the MRS for $B_{z}^{(+)}$and for $B_{z}^{(-)}$events, and for Quiet $B_{z}^{(+)}$and for Disturbed $B_{z}^{(-)}$events; in all such cases, no $B_{z}$ polarity change occurred before or around the time when the SI was thought to hit the magnetosphere. We do not show such plots here, but report that the curves for $B_{z}^{(+)}$and Quiet $B_{z}^{(+)}$are very similar to that for Quiet Is shown in Fig. 6, upper panel; similarly, the curves for $B_{z}^{(-)}$and Quiet $B_{z}^{(-)}$ resemble that for Disturbed Is shown in Fig. 6, lower panel. Such similarities are not surprising, as expected from the distribution of cases shown in Table 1 . We did not consider $B_{z}^{v a r}$ events, as our purpose was to exclude the cases when $B_{z}$ changed sign prior to or at the SI, and the Quiet $B_{z}^{(-)}$and Disturbed $B_{z}^{(+)}$events, since they are very few. In conclusion, 


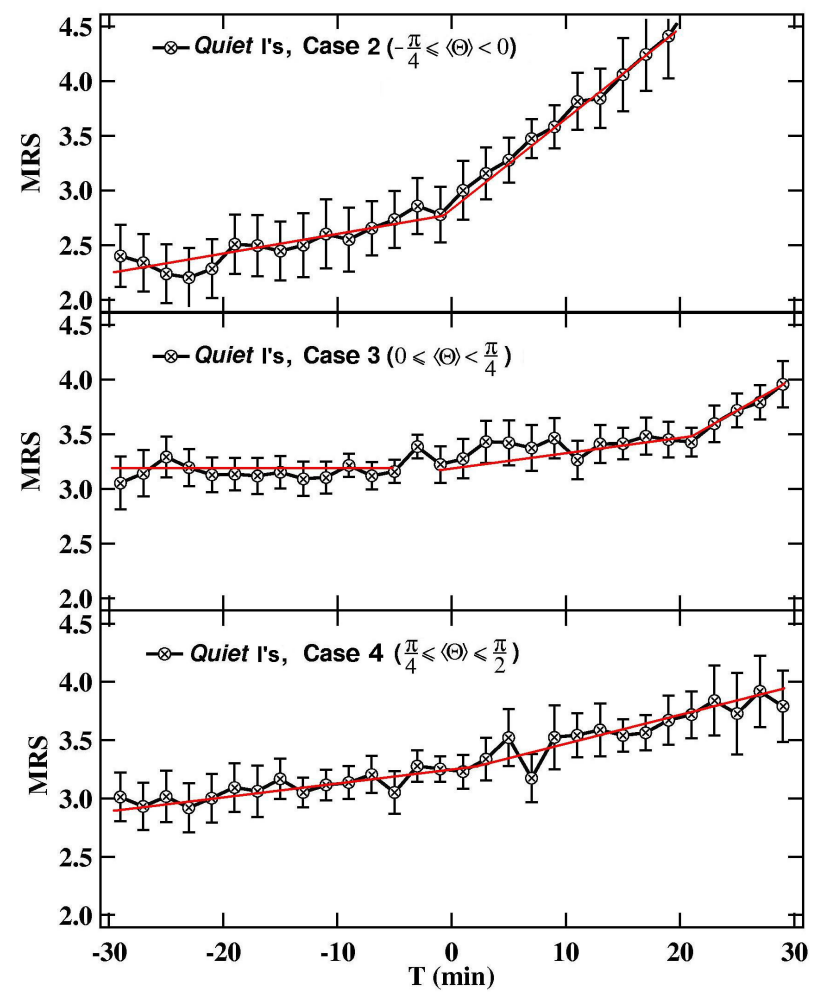

Fig. 7. Quiet Is classified according to $\langle\Theta\rangle$. Upper panel: plot of MRS vs. time for Case $2(-\pi / 4 \leq\langle\Theta\rangle<0,17$ events $)$; Middle panel: plot of MRS vs. time for Case $3(0 \leq\langle\Theta\rangle<\pi / 4,25$ events); Lower panel: plot of MRS vs. time for Case $4(\pi / 4 \leq\langle\Theta\rangle \leq \pi / 2$, 17 events).

we can state that the changes in MRS observed at the SIs are not a by-product of changes in $B_{z}$ occurring prior to SI or at the SI time.

In order to further investigate the effects of the IMF orientation, including also in the analysis the magnetic field $y$ component, we made a more quantitative classification of the events on the basis of the angle $\Theta=\arctan \left(B_{z} /\left|B_{y}\right|\right)$.

The average value of $\Theta$ was calculated over the same $[-30,10] \mathrm{min}$ interval around $T_{W I N D}$ that we used in the above $B_{z}$ classification. We built four classes as follows:

- Case 1: events for which $-\frac{\pi}{2} \leq\langle\Theta\rangle<-\frac{\pi}{4}$,

- Case 2: events for which $-\frac{\pi}{4} \leq\langle\Theta\rangle<0$,

- Case 3: events for which $0 \leq\langle\Theta\rangle<\frac{\pi}{4}$,

- Case 4: events for which $\frac{\pi}{4} \leq\langle\Theta\rangle \leq \frac{\pi}{2}$.

Table 2 summarizes the classification. The classes of Case 1 for Quiet Is, and of Cases 3, 4 for Disturbed Is include very few events, so that we do not discuss them further. MRS is then calculated for Quiet Is - Cases 2, 3, 4, and for Disturbed Is - Cases 1, 2 only.

Figure 7 shows MRS vs. time for Quiet Is, for three $\langle\Theta\rangle$ classes. The upper panel refers to Case 2 (17 events): the

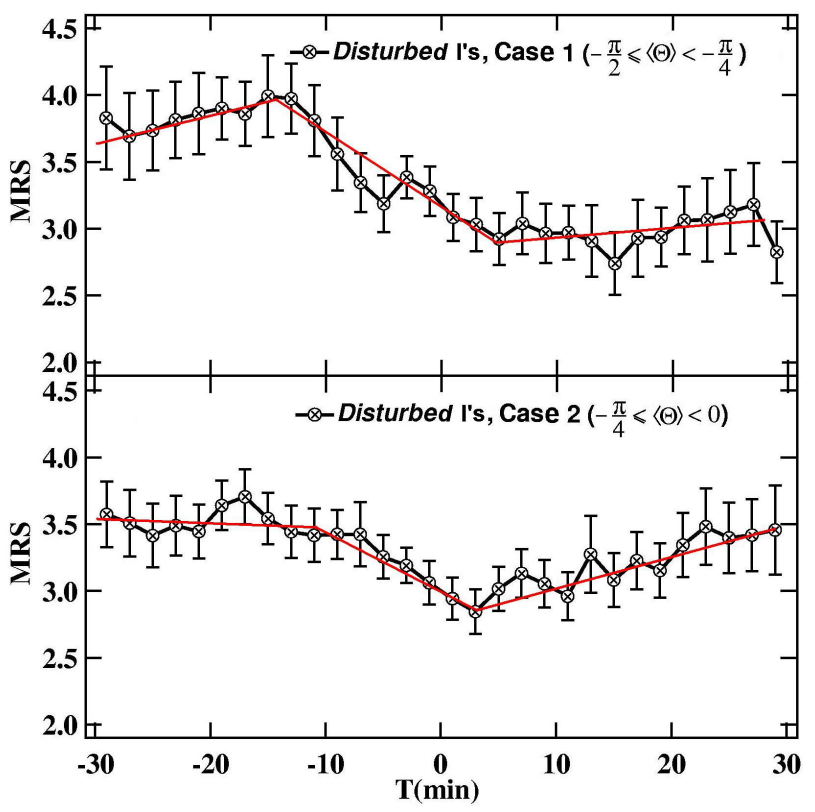

Fig. 8. Disturbed Is classified according to $\langle\Theta\rangle$. Upper panel: plot of MRS vs. time for Case $1(-\pi / 2 \leq\langle\Theta\rangle<-\pi / 4,14$ events); Lower panel: plot of MRS vs. time for Case $2(-\pi / 4 \leq\langle\Theta\rangle<0,26$ events).

Table 2. Number of Quiet and Disturbed Is for four subsets depending on $\langle\Theta\rangle$, i.e. the average value of $\Theta=\arctan \left(B_{z} /\left|B_{y}\right|\right)$, calculated over a $[-30,10]$ min interval around $T_{W I N D}$.

\begin{tabular}{cccc}
\hline Type & Tot & Quiet & Disturbed \\
\hline$-\pi / 2 \leq\langle\Theta\rangle<-\pi / 4$ & 15 & 1 & 14 \\
$-\pi / 4 \leq\langle\Theta\rangle<0$ & 43 & 17 & 26 \\
$0 \leq\langle\Theta\rangle<\pi / 4$ & 32 & 25 & 7 \\
$\pi / 4 \leq\langle\Theta\rangle \leq \pi / 2$ & 18 & 17 & 1 \\
\hline
\end{tabular}

MRS curve displays a rise in the trend in the whole interval, but around $T=0$ a remarkable change in slope is noticed, making the rise steeper than before. This effect is much less evident in the other two panels. In fact, for Case 3 (25 events, middle panel) $\mathrm{MRS} \simeq 3.2$ up to $T \simeq-4$, then a slight increase is observed, after which $\mathrm{MRS} \simeq 3.54$ up to $T \simeq 20 \mathrm{~min}$, when it increases again. The lower panel refers to Case 4 (17 events): a rise in the trend is seen in the whole period, with a slight change in slope around $T=0$. It appears clearly that the curve for Quiet Is, shown in Fig. 6, is obtained from the sum of the three cases just described.

Figure 8 shows MRS vs. time for Disturbed Is, for two $\langle\Theta\rangle$ classes. The upper panel refers to Case 1 (14 events): in the first part of the interval $(-30<T<-13 \mathrm{~min})$ MRS rises, then begins to fall, reaching a minimum around $T=5 \mathrm{~min}$, and keeping a constant behaviour afterward. The lower panel refers to Case 2 (26 events): MRS keeps a constant level up to $T \simeq-8 \mathrm{~min}$, then decreases up to $T \simeq 3 \mathrm{~min}$, and finally, rises again in the last part of the interval. These two curves resemble closely the curve for Disturbed Is, shown in Fig. 6. 

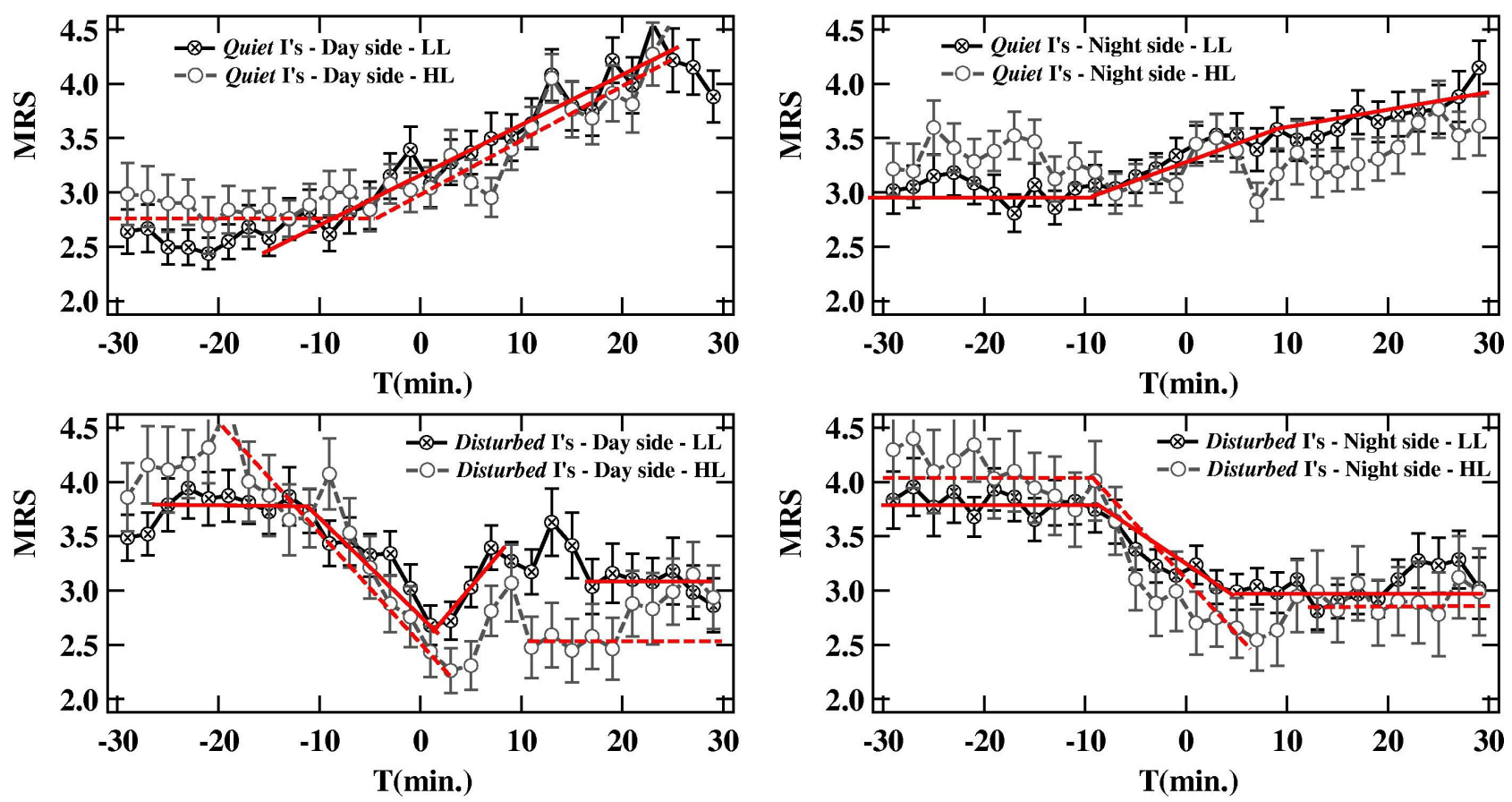

Fig. 9. Left side - Upper panel: plot of MRS vs. time for dayside (06:00-18:00 MLT) Quiet Is: the solid line refers to Low Latitude (LL, $60^{\circ}-70^{\circ} \Lambda$ ), while the dashed line refers to High Latitude (HL, $70^{\circ}-80^{\circ} \Lambda$ ). Left side - Lower panel: the same as for the upper panel, but for Disturbed Is. Right side - Upper panel: plot of MRS vs. time for nightside (18:00-06:00 MLT) Quiet Is: the solid line refers to Low Latitude $\left(\mathrm{LL}, 60^{\circ}-70^{\circ} \Lambda\right.$ ), while the dashed line refers to High Latitude (HL, $\left.70^{\circ}-80^{\circ} \Lambda\right)$. Right side - Lower panel: the same as for the upper panel, but for Disturbed Is.

Table 3. Number of echoes for Quiet and Disturbed Is depending on MLT and Magnetic Latitude. Dayside extends from 06:00 to 18:00 MLT, nightside extends from 18:00 to 06:00 MLT. LL stands for Low Latitude $\left(60^{\circ}-70^{\circ} \Lambda\right)$, while HL stands for High Latitude $\left(70^{\circ}-80^{\circ} \Lambda\right)$.

\begin{tabular}{ccccc}
\hline \multicolumn{2}{c}{ Number of Echoes: } & Tot & LL & HL \\
\hline \multirow{2}{*}{ Dayside } & Quiet & 387412 & 159672 & 227740 \\
& Disturbed & 349072 & 222796 & 126276 \\
Nightside & Quiet & 491352 & 264396 & 226956 \\
& Disturbed & 459704 & 364631 & 95073 \\
\hline
\end{tabular}

\subsection{MLT and Magnetic Latitude analysis}

Echoes are sorted according to Magnetic Local Time, as dayside echoes (06:00-18:00 MLT), and nightside echoes (18:00-06:00 MLT), and according to Magnetic Latitude as Low Latitude echoes (LL, $60^{\circ}-70^{\circ} \Lambda$ ) and High Latitude echoes $\left(\mathrm{HL}, 70^{\circ}-80^{\circ} \Lambda\right)$. The MLT calculation is made according to the AACGM model (Baker and Wing, 1989).

The results of this data sorting is displayed in Fig. 9 for Quiet and Disturbed events. In the left side, upper panel, the plot for Quiet Is in the dayside is shown: the solid curve refers to LL, while the dashed one refers to HL. The same notation is also kept in the other panels. At LL, after a short plateau, the MRS grows almost continuously in the $[-20,30]$ min interval, while at HL a flat behaviour is noticed up to $T \simeq-5 \mathrm{~min}$, followed by a rise in the trend, similar for LL. Both curves resemble well the Quiet Is curve shown in the upper panel of Fig. 6. In the left side, lower panel, the plot for Disturbed Is in the dayside is shown. The curves for LL (solid), and HL (dashed) look very similar, showing an MRS decrease starting between $T \simeq-15$ and $T \simeq-10 \mathrm{~min}$, and reaching a minimum at about $T \simeq 5 \mathrm{~min}$. Afterward a little increase is reported, possibly followed by a broad plateau. The fall of MRS seems to be more evident at HL. In the right side, upper panel, the plot for Quiet Is in the nightside is shown. Again, we can notice a rise in the trend for MRS at LL, starting from $T \simeq-8 \mathrm{~min}$, though less pronunced than in the dayside. At HL no clear changes can be outlined, because of the strong fluctuations. In the right side, lower panel, the plot for Disturbed Is in the nightside is shown. In both LL and HL curves, still one can see an MRS decrease starting at $T \simeq-8 \mathrm{~min}$. A lower level is reached at about $T \simeq 0$ and hence a plateau follows. As for the dayside, the decrease in MRS seems to be more important at HL.

In Table 3 the number of echoes for the different subsets is summarised. The order of magnitude is the same for the echoes of the Quiet and Disturbed subsets, and more echoes are observed during the night. This result has been already shown in Fig. 3. For Quiet events, on the dayside, the number of echoes is greater at HL than at LL; for Disturbed events, 
this number is greater at LL, on both day- and nightsides. Therefore, one can suggest that the nonnegligible amount of echoes shown in Fig. 3 between 09:00 and 15:00 MLT and $70^{\circ}-80^{\circ} \Lambda$, could derive from the Quiet Is.

\section{Discussion}

We start this section with a brief discussion of how we calculated the delay between the SI observation at WIND and its expected effects in the ionosphere. First of all, it must be said that the use of Eq. (1) will produce a small shift to positive $T$ of the SI effect. As a matter of fact, Eq. (1) implies using the undisturbed solar wind velocity for the propagation in the magnetosheath and in the magnetosphere. Neglecting here the small underestimate of $\Delta \tau$ due to the reduced magnetosheath speed, we recall that the propagation time between the magnetopause and the dayside ionosphere is usually estimated in 6-8 min (e.g. Lysak, 1986; Watanabe et al., 1986; Todd et al., 1988), to be compared with $\Delta \tau \simeq 4 \mathrm{~min}$ which is included in Eq. (1) for a $V_{x} \simeq 300 \mathrm{~km} / \mathrm{s}$ and an average magnetopause stand-off distance of $10 R_{E}$. In conclusion, on average, the underestimate produced by the use of Eq. 1 is expected to be around 2-4 min. This is comparable to the SuperDARN 2-min resolution, so that we do not try to introduce any correction to this regard. To further discuss the delay issue, we show in Figure 10 the $\operatorname{GSE}(X, Y)$ distribution of the WIND positions for all 178 events in the data set. Clearly one can see that from November 1997 to September 2000, the WIND position is affected by a large spread, both along the Sun-Earth direction, and along $Y$. About $60 \%$ of the events lies at $X>50 R_{E}$, and $40 \%$ at $X>100 R_{E}$; moreover, about $30 \%$ of the events had $|Y|>50 R_{E}$. It is expected that this will have an impact on the calculation of the delay through Eq. (1). To this regard, a recent work by Weimer et al. (2002), focuses the attention on the possible effects on the propagation time of the interplanetary discontinuities' orientation and of their evolution from L1 to the Earth's orbit, and quotes remarkable differences between the measured delays and those calculated as in Eq. (1). The cited authors also show how in some cases the correct propagation time can be correctly evaluated. Unfortunately, this procedure is cumbersome and cannot always be applied. As such, its use is not feasable for this study. To go back to the present issue, as an example of such an effect in our database, we recall that in the SI event of Fig. 2, the SI travel time to the Earth's ionosphere appeared to be $5 \mathrm{~min}$ lower than calculated. In other cases, the opposite occurs and the travel time appears to be larger than expected. Consequently, the effects must show up, for the various data sets used in the paper, as an apparent time spread of the SI effect on MRS, which is an average over RS of the individual events in the current set of events (see Eqs. (3) and (4)). Actually, this is shown by the description of all the plots of MRS vs. time made above: the MRS minima, maxima and level changes are frequently smeared out on time scales of several minutes. However, in spite of such uncertainties, these features are clearly recognizable:

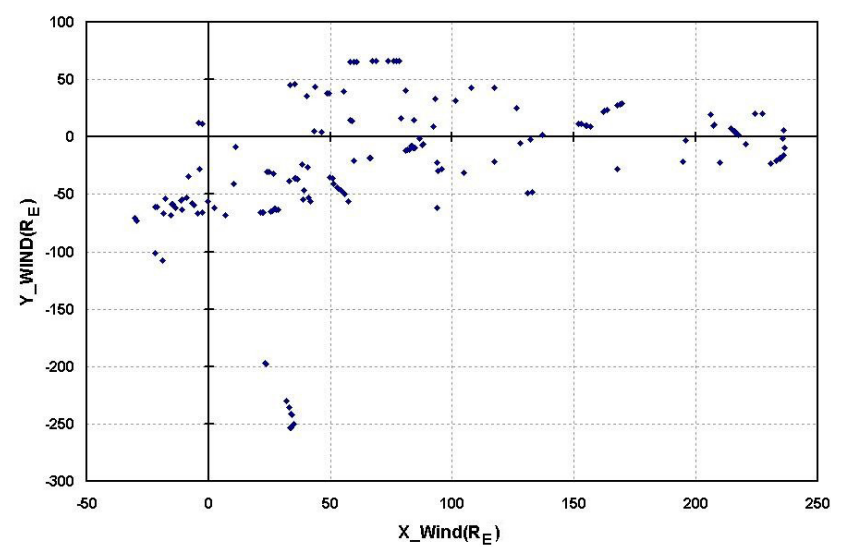

Fig. 10. WIND positions for all 178 events in the data set (November $1997 \rightarrow$ September 2000$)$ in the GSE $(X, Y)$ plane, in $R_{E}$ units.

in particular, the MRS level changes evidenced in the analysis performed in Sect. 4 often begin for $T<0$ and extend for $T>0$, but occur anyway around $T=0$; on the other hand, the maximum MRS value for Quiet events and the minimum value for Disturbed events, which we interpret as related to the SI, occur close to $T=0$ or a few minutes later (see, e.g. Fig. 4). To conclude this discussion on time delay, it appears that the main features of the MRS plots described in Sect. 4 can be interpreted as related to the SIs.

For a better understanding of the observed MRS behaviours, it is necessary to discuss at some length the processes responsible for the backscatter of radio waves. A signal of given wavelength $\lambda$ which propagates in the ionosphere is scattered only if it encounters a density fluctuation, usually created by plasma instabilities in the ionosphere. If $\lambda_{i r r} \simeq \lambda / 2$, where $\lambda_{i r r}$ is the irregularity wavelength, the signal is backscattered (Bragg condition). Such irregularities can be found in the $\mathrm{E}$ and $\mathrm{F}$ regions of the ionosphere, their wave vector $k_{i r r}$ being perpendicular to the geomagnetic field lines. Moreover, at the SuperDARN frequencies, the ionospheric refraction yields that the $k$ vectors of the transmitted waves are parallel to $k_{i r r}$ at the $\mathrm{E}$ and F-region altitudes, thus making backscatter possible (e.g. Hanuise et al., 1981). The dominant mechanism to produce such decameter scale irregularities in the F region is the Gradient Drift Instability (GDI). For complete reviews on ionospheric irregularities one can see Fejer and Kelley (1980), Keskinen and Ossakow (1983), Tsunoda (1988). Early HF radar observations and in-situ measurements, showed that a power law exists in the irregularity spectra, ranging from scales of hundreds of kilometers, down to meters (Villain et al., 1986). Many works in the literature seem to confirm this characteristic feature of the ionospheric irregularities: when a large-scale gradient is present in a given region, irregularities in a wide scale spectrum (from kilometers to meters) are also observed in the same site (Tsunoda, 1988 and ref. therein; Moen et al., 2002). This suggests that large-scale gradients give rise to a cascade process, generating smaller scale irregularities, though the actual physical mechanisms responsible for the 
irregularity production would not be necessarily the same at every scale. One of the most powerful mechanisms giving rise to large-scale gradients ( $100 \mathrm{~km}$ or more) is soft particle precipitation (energies of about a few tens of eV; e.g. Tsunoda, 1988). Moen et al. (2002) make a comparison between radio tomographic images of the ionosphere above Svalbard, meridian scanning photometer (MSP) and all-sky camera observations, and the echo occurrence in the field of view of the Hankasalmi SuperDARN radar. They find that the appearence of a large-scale density gradient $(\sim 100 \mathrm{~km})$ is well correlated, spatially and temporally, with a strong echo activity, which requires the presence of decameter-scale irregularities. Moreover, the MSP shows a 630-nm auroral emission, of a kind often associated with soft electron precipitation in the $\mathrm{F}$ region. They conclude that an enhanced electron precipitation may have produced the observed gradients and irregularities. Recent works by Milan et al. (2001) and Samson et al. (2003) show how a time series of quasi-periodical auroral forms can be related to characteristic frequencies of Field Line Resonances (FLR), often associated with Alfvén wave propagation along the geomagnetic field lines. Zhou and Tsurutani (1999) and Liou et al. (2002) argue that an increase in the solar wind ram and static pressure causes an increase in the perpendicular kinetic energy of trapped magnetospheric electrons and protons, which in turn induces the loss cone instability and the consequent particle precipitation in the ionosphere. Another mechanism which can be invoked for the decameter-scale irregularity growth in the F layer is related to the evolution in space and time of the so-called polar patches (e.g. Weber et al., 1984; Tsunoda, 1988). The patches are large-scale plasma structures $(\sim 1000 \mathrm{~km})$ which often can be found in the polar cap, mostly during periods of southward IMF $B_{z}$ or $K_{p}>4$. They are thought to originate in the dayside auroral oval by solar ionisation, and to drift antisunward through the polar cap with speeds of 250 to $700 \mathrm{~m} / \mathrm{s}$. When the IMF $B_{z}$ turns southward the auroral oval expands to lower latitudes, a two-cell configuration of the plasma convection is established, and the convection velocity increases considerably: patches are thus convected towards the polar cap where they can be detached from following mid-latitude plasma (Tsunoda, 1988). There are recent experimental evidences of small-scale structuring in patches (Basu et al., 1995; Basu and Valladares, 1999): a remarkable similarity is observed between the spatio-temporal characteristics of the polar cap scintillation caused by mesoscale irregularities ( $1 \mathrm{~km}$ or less), and a model prediction of macroscale structures under southward IMF $B_{z}$ conditions. This result implies that the mesoscale structures causing scintillation of satellite signals often are formed at the edges of polar cap patches through the gradient drift instability process and permeate the entire patch.

Let us now introduce the discussion of our results. Firstly, it is to say that we see a global effect of SIs on the radar echoes, especially for Is. The effect looks like a broad depression of the MRS followed by a rise, as described in Sect. 4 and shown in Fig. 4. This effect is a superposition of two different behaviours, mainly driven by the different geomagnetic conditions, as the selection made in Sect. 4.1, according to $\mathrm{AE}$, seems to suggest.

For what concerns Quiet events, a growth of MRS is seen in correspondence with the SI time (see Fig. 6, upper panel) for both I and D events. The similar behaviour for Is and Ds could confirm Araki's prediction (Araki, 1994), that both positive and negative SIs cause the same effects in the magnetosphere, with the exception for the sense of FACs. However, this result must be taken with care, as the visual inspection of all single Quiet D events suggests that they display a larger variability than the Quiet I events; moreover, the smaller number of $\mathrm{D}$ events does not allow their analysis as a function of IMF, MLT and magnetic latitude.

The latitudinal analysis for Quiet Is made in Subsect. 4.3 (see Fig. 9), evidences that MRS shows a clear response to SIs both at low and high latitudes, though the number of echoes is smaller at lower latitudes. We propose the following interpretation: when the pressure pulse hits the magnetopause, a fast, compressional MHD wave is launched, as described in Sect. 1, and couples to a shear Alfvén wave at a resonance position which depends on the local Alfvén speed (Samson et al., 2003); this results in a FLR which induces soft particle precipitation in the $\mathrm{F}$ region and a consequent increase in the ionospheric irregularities and of MRS. It is reasonable to expect that such a mechanism be more effective at lower latitudes, where the footprint of the Central Plasma Sheet (CPS) usually maps $\left(\sim 65^{\circ}-70^{\circ} \Lambda\right)$. On the other hand, the precipitation of trapped electrons, due to loss cone instability induced by the SI in the magnetosphere, can also account for the lower latitude MRS increase. Regarding the effect at higher latitudes, it is necessary to invoke an additional mechanism. For that purpose, we recall that Newell and Meng (1994) studied the variation of the ionospheric footprints of the magnetospheric regions as a function of the solar wind dynamic pressure, through the observation of precipitation data from DMSP satellites; they found that the high latitude $\left(\sim 75^{\circ}-82^{\circ} \Lambda\right)$ footprints of magnetospheric Cusp and Low Latitude Boundary Layer (LLBL), are limited to a small latitudinal range when the pressure is low $(p \leq 2 \mathrm{nPa})$ and cover the whole $70^{\circ}-80^{\circ} \Lambda$ range for $p \geq 4 \mathrm{nPa}$. They concluded that this effect is only due to the pressure value and suggested that impulsive solar wind penetration in the Cusp and LLBL could be favoured by a high solar wind dynamical pressure and cause particle precipitation along magnetic field lines. Moreover, recent radar observations seem to confirm that most high latitude echoes come from the ionospheric Cusp (e.g. Villain et al., 2002). In conclusion, we suggest that the expansion of the Cusp and LLBL footprints between $70^{\circ}$ and $80^{\circ} \Lambda$ and the associated particle precipitation could account for the observed high-latitude MRS growth at the SI time.

Let us now move to the discussion of MRS for Disturbed events. First of all, it must be said that the Disturbed events' set is far less homogeneous than the Quiet events' set. This stems from its definition and is confirmed by visual inspection of the solar wind parameters and AE. Therefore, the results pertaining to this event set must be considered with 
care. This is even more so regarding the Disturbed Ds subset and we shall not discuss the broad peak seen for the Disturbed Ds around $T=0$ in the lower panel of Fig. 6. Regarding the I events, the most impressive feature to be noticed is a broad depression of echoes around $T=0$. A radio signal absorption during substorms or, more generally, during Sudden Commencements (SC), is a well-known fact in the riometer community. Ortner et al. (1962) analyzed data from 25 riometer stations, located in the Northern Hemisphere between $56.4^{\circ}$ and $88^{\circ} \Lambda$, for $71 \mathrm{SC}$ occurring between 1958 and 1960. They found a good correlation between riometer and magnetometer records taken in the same site: the riometer diagrams clearly showed a strong absorption in coincidence with SC's. Moreover they noticed that the absorption was maximum for stations located near the maximum of the auroral activity region, following the Feldstein model of the auroral oval (Feldstein, 1960). They interpreted this in terms of loss cone electron precipitation from the inner magnetosphere, due to the modified geometry of the geomagnetic field lines during a SC. But how could the same precipitation induce an increase in MRS for Quiet events, and a decrease for Disturbed events? We can suppose that, if an SI occurs during periods of high geomagnetic activity, the particle precipitation induced by the SI is superimposed to precipitation induced by other phenomena, and will be globally stronger and with a wider energy spectrum than for a Quiet event. This is also confirmed by observations of Kavanagh et al. (2002), who discuss an SI occurring at 06:50 UT on 11 February 1997 during the recovery phase of a substorm. At the SI time and prior to it, they report high values of the AE index, such that, according to our criteria, this would be classified as a Disturbed event. At the same time they report high electron precipitation rates with energy $>2 \mathrm{keV}$ above northern Scandinavia. If such precipitation is a common feature of Disturbed events, it is worth noticing that, at energies higher than $1 \mathrm{keV}$, electrons are not completely stopped by the $\mathrm{F}$ region and can largely penetrate to the $\mathrm{E}$ and $\mathrm{D}$ regions. This particle precipitation and the consequent increase in the plasma density, is responsible for two main effects on HF radio signals: 1) an enhanced absorption in the $\mathrm{D}$ region of the ionosphere, and 2) an enhanced refraction in the $\mathrm{E}$ region, which impedes the propagation to the $\mathrm{F}$ region. The combination of the two effects can result in a more pronounced decrease in the number of echoes observed at higher latitudes, between $70^{\circ}$ and $80^{\circ} \Lambda$. This is actually seen in our data, as shown in Fig. 9. At lower latitudes the perpendicularity condition is more easily satisfied and the effects on MRS is globally less important.

Regarding the MLT dependence of MRS, we recall that very clear trends are seen for both Quiet and Disturbed Is in the dayside, while in the nightside the SI effect seems to be somewhat reduced (Fig. 9). To this respect, it can be noted that SIs are expected to hit the magnetopause mainly in the dayside, so that the night effect could be smaller and possibly delayed. On the other hand, on the nightside the SI effects could be masked by a higher echo activity as shown by the echo numbers listed in Table 3. In fact, SuperDARN measurements often show a higher level of echoes during the night (e.g. Ruohoniemi and Greenwald, 1997; Milan et al., 1997), when the irregularity growth rate is higher; on the contrary, during the day the solar photoionisation acts as a major constraint on the GDI formation, as the ionospheric density increases globally and uniformly, and the density gradients are smoothed down.

As it has been reported in Sect. 4, the MRS behaviour for the $B_{z}^{(+)}$subset of Quiet events is very similar to that in the whole set of Quiet Is. The same applies to the $B_{z}^{(-)}$subset in relation to the Disturbed events set. In fact, this is expected, because a general correlation between the IMF orientation and the $\mathrm{AE}$ index behaviour has been well known for a long time (e.g. Arnoldy, 1971; Akasofu, 1979). Akasofu (1979) introduced the quantity $\varepsilon(t)=V B^{2} \sin ^{4}(\theta / 2) l_{0}^{2}$, where $B$ is the magnitude of IMF, $V$ is the solar wind speed, $\theta$ is the polar angle of the IMF vector in the $y-z$ plane in GSM coordinates and $l_{0} \simeq 7$ Earth radii. He showed that $\varepsilon(t)$ is actually the energy flux transferred from solar wind to the magnetosphere-ionosphere system, and he verified a good correlation with $\mathrm{AE}$ through the observation of several substorms. Regarding radar observations, Ballatore et al. (2001) performed a statistical study on SuperDARN echoes over a long period of time, and found a good correlation between the rate of scattering and $\varepsilon(t)$. We can thus conclude that the mean level of geomagnetic activity is surely influenced by the IMF orientation, but our results indicate that the solar wind dynamic pressure itself leads to clear effects on the MRS, in both situations of northward and southward $B_{z}$. This picture is substantially confirmed by the selection of the events on the basis of the angle $\Theta=\arctan \left(B_{z} /\left|B_{y}\right|\right)$. For what concerns Quiet events (see Fig. 7), an increase in MRS in correspondence with the SI is always reported, for every configuration of IMF. When $\pi / 4 \leq\langle\Theta\rangle \leq \pi / 2\left(B_{z}\right.$ strongly positive and dominant on $\left.B_{y}\right)$, the MRS growth is smaller, though beyond the error bars. Actually, also when $0 \leq\langle\Theta\rangle<\pi / 4\left(B_{z}\right.$ positive but $B_{y}$ dominant on $B_{z}$ ), the MRS behaviour does not look very different from the previous case: this leads us to think that the $B_{y}$ component plays a marginal role with respect to $B_{z}$, during an SI occurrence. Moreover, looking at the Quiet events with $-\pi / 4 \leq\langle\Theta\rangle<0\left(B_{z}\right.$ negative, $B_{y}$ dominant on $\left.B_{z}\right)$, we report an MRS rise much steeper than for the other cases. We can conclude that $B_{z}$ has possibly an effect on the echo level, during an SI event: if $B_{z}$ is southward in the period that preceeds the discontinuity, the compression of the magnetosphere due to an SI more easily induces particle precipitation in the high latitude ionosphere, with the mechanisms described above. Moreover, the southward $B_{z}$ configuration can be related to the presence of patches in the polar cap, which could lead to an increase in decameter-scale irregularities. The Disturbed events seem not to be sensibly influenced by $\Theta$ (see Fig. 8): most of these events fall into cases with $\langle\Theta\rangle<0$, and an MRS decrease is always observed, having more or less the same features in both the considered configurations $(-\pi / 2 \leq\langle\Theta\rangle<-\pi / 4,-\pi / 4 \leq\langle\Theta\rangle<0)$. 


\section{Summary and conclusions}

In this work $236 \mathrm{SI}$ events were examined to investigate their effects on SuperDARN HF radar echoes. After excluding part of them on the basis of statistical considerations, as discussed in Sect. 3, the final analysis was performed on a set of 178 events, 114 Increases (I) and 64 Decreases (D) of the solar wind dynamic pressure. The analysis was based on the calculation of averages of the Mean Rate of Scattering (MRS) for thirty 2-min bins around the SI time, over all radars and over various data subsets. The response of the ionosphere to the SIs, although global in nature, depends on various conditions, so that when averaged over the whole data set, results to be the combination of diverse effects. For this reason, MRS averages were calculated for various subsets of data, depending on the AE index, IMF orientation, MLT, magnetic latitude, SI polarity. The main results are summarized hereafter.

1. Positive and negative SIs. Both sudden increases (I) and decreases (D) of the solar wind dynamic pressure affect the SuperDARN echoes. In the case of I (D) events the MRS displays a decrease (increase) roughly around with the SI. The decrease for I events results from a superposition of different effects, as detailed hereafter. On the other hand, the smaller statistics of the D cases does not allow for a similar detailed investigation.

2. Quiet I events. Quiet Is display an increase in MRS close to $T=0$. This is seen both at LL and at HL. At lower latitudes we suggest that soft electrons can precipitate to the ionosphere due to loss cone instability induced by the SI or to FLRs generated by the SI related Alfvén waves; this precipitation in the $\mathrm{F}$ region induces density gradient formation, thus enhancing the GDI growth rate and increasing the number of echoes. At higher latitudes, the MRS increase may be caused by particle precipitation related to the enlargement of the cusp and LLBL regions.

3. Disturbed I events. Disturbed Is display a large dip of MRS around $T=0$ at HL and a smaller dip at LL. Again, SIs cause particle precipitation due to FLR's and loss cone effects, as described in point 2 , but now the geomagnetic activity is higher and the precipitation stronger and with a wider energy spectrum, thus allowing particle penetration down to the $\mathrm{D}$ and $\mathrm{E}$ regions. Two concurrent effects are thought to take place: an enhanced absorption of the radio signal in the $\mathrm{D}$ region and a strong refraction in the $\mathrm{E}$ region, impeding the propagation to the $\mathrm{F}$ region, mainly at higher latitudes.

4. MLT effects. The general behaviours described in points 2 and 3 for Quiet and Disturbed Is show up more clearly in the MLT dayside (06:00-18:00 MLT) than on the nightside. This can be explained by the consideration that on the nightside the SI effect is generally thought to be smaller and can be masked by a large background echo activity.
5. The role of IMF. From the analysis made under various IMF $B_{z}$ conditions, we concluded that the effects herein summarized are due to the SI itself and not specifically driven by changes in IMF $B_{z}$. However, $B_{z}$ has possibly an effect on the echo level during an SI event: if $B_{z}$ is southward in the period that preceeds the discontinuity, the compression of the magnetosphere seems to result in a larger MRS increase that can be attributed to enhanced particle precipitation in the high latitude ionosphere. On the contrary, $B_{y}$ does not seem to play a noticeable role to this regard.

Acknowledgements. The authors would like to thank WDC for Geomagnetism, Kyoto, for the use of the AE index data; R. Lepping and K. Ogilvie for the use of WIND magnetic field and plasma data; the CDAWeb team; the principal investigators of the SuperDARN radars whose data have been used in this work: R. A. Greenwald (Goose Bay and Kapuskasing), M. Lester (Hankasalmi and Thykkvibær), G. Sofko (Saskatoon). Finally, the authors thank one of the referees for his/her valuable comments and suggestions.

Topical Editor M. Lester thanks S. Milan and another referee for their help in evaluating this paper.

\section{References}

Akasofu, S. -I.: Interplanetary energy flux associated with magnetospheric substorms, Planet. Space Sci., 27, 425-431, 1979.

Araki, T.: A physical model of the geomagnetic sudden commencement, in Solar Wind Sources of Magnetospheric Ultra-LowFrequency Waves, Geophys. Monogr. Ser., 81, 183-200, 1994.

Arnoldy, R. L.: Signature in the Interplanetary Medium for Substorms, J. Geophys. Res., 76, 5189-5200, 1971.

Baker, K. B., and Wing, S.: A new magnetic coordinate system for conjugate studies at high latitudes, J. Geophys. Res., 94, 91399143, 1989.

Ballatore, P., Villain, J.-P., Vilmer, N., and Pick, M.: The influence of the interplanetary medium on SuperDARN radar scattering occurrence, Ann. Geophys., 18, 1576-1583, 2001,

SRef-ID: 1432-0576/ag/2000-18-1576.

Basu, Sa., Basu, Su., Sojka, J. J., Schunk, R. W. and MacKenzie, E.: Macroscale modeling and mesoscale observations of plasma density structures in the polar cap, Geophys. Res. Lett., 22, 881884, 1995.

Basu, Sa. and Valladares, C.: Global aspects of plasma structures, J. Atmos. Solar-Terr. Phys., 61, 127-139, 1999.

Davis, T. N. and Sugiura, M.: Auroral electrojet activity index AE and its universal time variations, J. Geophys. Res., 71, 785, 1966.

Fejer, B. G. and Kelley, M. C.: Ionospheric irregularities, Rev. Geophys. Space Phys., 18, 401-454, 1980.

Feldstein, Y. I.: Geographical distribution of the aurora and azimuth of auroral arcs, Investigation of the aurora, Acad. Sci. USSR, 4, $61,1960$.

Friis-Christensen, E., McHenry, M. A., Clauer, C. R., and Vennerstrøm, S.: Ionospheric traveling convection vortices observed near the polar cleft: A triggered response to sudden changes in the solar wind, Geophys. Res. Lett., 15, 253-256, 1988.

Greenwald, R. A., Baker, K. B., Dudeney, J. R., Pinnock, M., Jones, T. B., Thomas, E. C., Villain, J.-P., Cerisier, J.-C., Senior, C., Hanuise, C., Hunsucker, R. D., Sofko, G., Koehler, J., Nielsen, E., Pellinen, R., Walker, A. D. M., Sato, N., and Yamagishi, H.: 
DARN/SUPERDARN: A Global View of the Dynamics of HighLatitude Convection, Space Sci. Rev., 71, 761-796, 1995.

Hanuise, C., Villain, J.-P., and Crochet, M.: Spectral studies of Fregion irregularities in the auroral zone, Geophys. Res. Lett., 8, 1083-1086, 1981.

Hanuise, C., Greenwald, R. A., and Baker, K. B.: Drift motions of very high latitude $F$ region irregularities: Azimuthal Doppler analysis, J. Geophys. Res., 90, 9717-9725, 1985.

Kavanagh, A. J., Honary, F., McCrea, I. W., Donovan, E., Woodfield, E. E., Manninen, J., and Anderson, P. C.: Substorm related changes in precipitation in the dayside auroral zone - a multi instrument case study, Ann. Geophys., 20, 1321-1334, 2002, SRef-ID: 1432-0576/ag/2002-20-1321.

Keskinen, M. J. and Ossakow, S. L.: Theories of high-latitude ionospheric irregularities: A review, Radio Sci., 18, 1077-1091, 1983.

Lepping, R. P., Acuna, M. H., Burlaga, L. F., Farrell, W. M., Slavin, J. A., Schatten, K. H., Mariani, F., Ness, N. F., Neubauer, F. M., Whang, Y. C., Byrnes, J. B., Kennon, S., Panetta, P. V., Scheifele, J., and Worley, E. M.: The Wind Magnetic Field Investigation, Space Sci. Rev., 71, 207-229, 1995.

Liou, K., Wu, C.-C., Lepping, R. P., Newell, P. T., and Meng, C.-I.: Midday sub-auroral patches (MPSs) associated with interplanetary shocks, Geophys. Res. Lett., 29(16), doi:10.1029/2001GL014182, 2002.

Lysak, R. L.: Coupling of the dynamic ionosphere to auroral flux tubes, J. Geophys. Res., 91, 7047-7056, 1986.

Milan, S. E., Yeoman, T. K., Lester, M., Thomas, E. C., and Jones, T. B.: Initial backscatter occurrence statistics from the CUTLASS HF radars, Ann. Geophys., 15, 703-718, 1997, SRef-ID: 1432-0576/ag/1997-15-703.

Milan, S. E., Sato, N., Ejiri, M., and Moen, J.: Auroral forms and the field-aligned current structure associated with field line resonances, J. Geophys. Res., 106, 25 825-25 833, 2001.

Moen, J., Walker, I. K., Kersley, L., and Milan, S. E.: On the generation of cusp HF backscatter irregularities, J. Geophys. Res., 107(A4), doi:10.1029/2001JA000111, 2002.

Newell, P. T. and Meng, C.-I.: Mapping the dayside ionosphere to the magnetosphere according to particle precipitation characteristics, Geophys. Res. Lett., 19, 609-612, 1992.

Newell, P. T. and Meng, C.-I.: Ionospheric projection of magnetospheric regions under low and high solar wind pressure conditions, J. Geophys. Res., 99, 273-286, 1994.

Ogilvie, K. W., Chornay, D. J., Fritzenreiter, R. J., Hunsaker, F., Keller, J., Lobell, J., Miller, G., Scudder, J. D., Sittler Jr., E. C., Torbert, R. B., Bodet, D., Needell, G., Lazarus, A. J., Steinberg, J. T., Tappan, J. H., Mavretic, A., and Gergin, E.: SWE: A Comprehensive Plasma Instrument for the Wind Spacecraft, Space Sci. Rev., 71, 55-77, 1995.

Ortner, J., Hultqvist, B., Brown, R. R., Hartz, T. R., Holt, O., Landmark, B., Hook, J. L., and Leinbach, H.: Cosmic Noise Absorption Accompanying Geomagnetic Storm Sudden Commencements, J. Geophys. Res., 67, 4169-4186, 1962.

Ruohoniemi, J. M. and Greenwald, R. A.: Rates of scattering occurrence in routine HF radar observations during solar cycle maximum, Radio Sci., 32, 1051-1070, 1997.

Russell, C. T., Ginskey, M., and Angelopoulos V.: Effect of sudden impulses on currents in the auroral ionosphere under northward interplanetary magnetic field conditions: A case study, J. Geophys. Res., 99, 17 617-17 622, 1994.
Samson, J. C., Rankin, R., and Tikhonchuk, V. T.: Optical signatures of auroral arcs produced by field line resonances: comparison with satellite observations and modeling, Ann. Geophys., 21, 933-945, 2003,

SRef-ID: 1432-0576/ag/2003-21-933

Sibeck, D. G.: A Model for the Transient Magnetospheric Response to Sudden Solar Wind Dynamic Pressure Variations, J. Geophys. Res., 95, 3755-3771, 1990.

Sibeck, D. G., Trivedi, N. B., Zesta, E., Decker, R. B., Singer, H. J., Szabo, A., Tachihara, H., and Watermann, J.: Pressure-pulse interaction with the magnetosphere and the ionosphere, J. Geophys. Res., 108(A2), doi:10.1029/2002JA009675, 2003.

Sitar, R. J., Clauer, C. R., and Friis-Christensen, E.: High-latitude ground-based response to sudden changes in solar wind dynamic pressure, J. Geophys. Res., 101, 27 001-27 013, 1996.

Southwood D. J. and Kivelson, M. G.: The magnetohydrodynamic response of the magnetospheric cavity to changes in solar wind dynamic pressure, J. Geophys. Res., 95, 2301-2309, 1990.

Takeuchi, T., Araki, T., Luehr, H., Rasmussen, O., Watermann, J., Milling, D. K., Mann, I. R., Yumoto, K., Shiokawa, K., and Nagai, T.: Geomagnetic negative sudden impulse due to a magnetic cloud observed on May 13, 1995, J. Geophys. Res., 105, 18 835$18846,2000$.

Thorolfsson, A., Cerisier, J.-C., and Pinnock, M.: Flow transients in the postnoon ionosphere: The role of solar wind dynamic pressure, J. Geophys. Res., 106, 1887-1901, 2001.

Todd, H. S., Cowley, W. H., Lockwood, M., Willis, D. M., and Luhr, H.: Response time of the high-latitude dayside ionosphere to sudden changes in the north-south component of the IMF, Planet. Space Sci., 36(12), 1415-1428, 1988.

Tsunoda, R. T.: High-Latitude F region irregularities: a review and syntesis, Rev. Geophys., 26, 719-760, 1988.

Villain, J.-P., Beghin, C., and Hanuise, C.: ARCAD3-SAFARI coordinated study of auroral and polar F-region ionospheric irregularities, Ann. Geophys., 4, 61-68, 1986.

Villain, J.-P., Greenwald, R. A., Baker, K. B., and Ruohoniemi, J. M.: HF Radar Observations of $E$ Region Plasma Irregularities Produced by Oblique Electron Streaming, J. Geophys. Res., 92, 12 327-12 342, 1987.

Villain, J.-P., André, R., Pinnock, M., Greenwald, R. A., and Hanuise, C.: A Statistical study of the Doppler spectral width of high-latitude ionospheric F-region echoes recorded with SuperDARN coherent HF radars, Ann. Geophys., 20, 1769-1781, 2002,

SRef-ID: 1432-0576/ag/2002-20-1769.

Watanabe, K. M., Ashour-Abdalla, M., and Sato, T.: A numerical model of magnetosphere-ionosphere coupling: Preliminary results, J. Geophys. Res., 91, 6973-6978, 1986.

Weber, E. J., Buchau, J., Moore, J. G., Sharber, J. R., Livingston, R. C., Winningham, J. D., and Reinisch, B. W.: $F$ Layer Ionisation Patches in the Polar Cap, J. Geophys. Res., 89, 1683-1694, 1984.

Weimer, D. R., Ober, D. M., Maynard, N. C., Burke, W. J., Collier, M. R., McComas, D. J., Ness, N. F., and Smith, C. W.: Variable time delay in the propagation of the interplanetary magnetic field, J. Geophys. Res., 107(A12), doi:10.1029/2001JA009102, 2002.

Zhou, X. and Tsurutani, B. T.: Rapid intensification and propagation of the dayside aurora: Large scale interplanetary pressure pulses (fast shocks), Geophys. Res. Lett., 26, 1097-1100, 1999. 